\title{
Excessive grooming induced by somatostatin or its analog SMS 201-995
}

\author{
Tjeerd B. Van Wimersma Greidanus *, Carla Maigret and Brigitte Krechting \\ Rudolf Magnus Institute for Pharmacology, Medical Faculty, University of Utrecht, \\ Vondellaan 6, 3521 GD Utrecht, The Netherlands
}

Received 10 July 1987, revised MS received 12 August 1987, accepted 22 September 1987

Intracerebroventricular (i.c.v.) administration of somatostatin or SMS 201-995 induces excessive grooming behavior in rats. The grooming inducing effect of somatostatin is rather weak, as doses of $300 \mathrm{ng}$ or less did not result in increased total grooming scores. In contrast a dose of $10 \mathrm{ng}$ SMS 201-995 already significantly increased the total grooming scores. However, doses of $100 \mathrm{ng}$ and more did not further increase the total grooming scores reached with a $50 \mathrm{ng}$ dose of this peptide. Systemic administration of SMS 201-995 in doses up to $900 \mu \mathrm{g}$ did not result in excessive grooming behavior. The patterns of excessive grooming induced by i.c.v. SMS 201-995 and somatostatin were characterized by a predominant display of scratching. Since peptide-induced scratching is mainly due to activation of opiate receptor systems it is suggested that opiate receptors are involved in the behavioral response to SMS 201-995 and somatostatin administration. This suggestion is further supported by the suppressive effect of naloxone on excessive grooming induced by these peptides. Haloperidol and neurotensin also suppress the excessive grooming induced by somatostatin but not that induced by SMS 201-995. Finally, tolerance developed to the grooming-inducing effect of SMS 201-995 and somatostatin. In addition there was cross tolerance between somatostatin and SMS 201-995.

Somatostatin; SMS 201-995; Grooming behavior

\section{Introduction}

In addition to its well known inhibitory effect on growth hormone $(\mathrm{GH})$ release from the pituitary, somatostatin has a variety of effects on the central nervous system (Plotnikoff et al., 1974; Renaud et al., 1975; Garcia-Sevilla et al., 1978). One of these effects is a potent excitatory action resulting in a profound reduction of slow wave sleep and REM sleep (Dodd and Kelly, 1978; Havlicek et al., 1976). Somatostatin also inhibits the extinction of active avoidance behavior, probably due to increased selective attention to the conditioned stimulus (Vécsei et al., 1987). Moreover, central administration of somatostatin in-

* To whom all correspondence should be addressed. duces motor excitation and an increase in stereotyped behavior which includes compulsory scratching and circular movements (Havlicek et al., 1976). Catatonia, seizures and barrel rotation are sometimes observed after intracerebroventricular (i.c.v.) administration of higher doses of somatostatin (Havlicek et al., 1976; Burke and Fahn, 1983). Intrathecal administration of somatostatin elicits dose-dependent biting, licking, hind limb scratching, writhing and retching in rats, with scratching as the most prominent response (Seybold et al., 1982). Scratching behavior has also been observed in mice after intracranial administration of somatostatin (Dobry et al., 1981). Thus, somatostatin appears to be a peptide that induces behavioral effects including excessive grooming/ scratching behavior after central administration. In contrast, the analog $\left[\mathrm{D}-\operatorname{Trp}^{8}\right]$ somatostatin does 
not induce excessive grooming, although higher doses of this peptide elicit barrel rotation in rats (Dokas et al., 1983).

The excessive grooming induced by various different peptides is of a different nature. ACTH induces excessive grooming in which the various grooming elements are more or less equally involved (Gispen and Isaacson, 1981; Spruijt and Gispen, 1983: Van Wimersma Greidanus et al., 1985a; 1986; in press) whereas the excessive grooming induced by bombesin is completely different, with excessive scratching as the most pronounced element (Van Wimersma Greidanus et al., 1985a,b; 1987; Spruijt et al., 1986b). $\beta$-Endorphin-induced excessive grooming is also characterized by a predominant display of scratching (Aloyo et al., 1983; Spruijt et al., 1986a; Van Wimersma Greidanus et al., in press). In addition it has been suggested that dopamine neurotransmitter systems are generally involved in peptideinduced grooming as modulators (Cools et al., 1978; Spruijt et al., 1986a), whereas opiate receptor systems are primarily involved in peptide-induced excessive scratching (Van Wimersma Greidanus et al., 1985b; 1986; in press).

A recently developed octapeptide analog of somatostatin, SMS 201-995 (Sandostatin ${ }^{\circledR}$ ), appears to have a very potent and long-acting inhibitory effect on growth hormone secretion (Bauer et al., 1982; Lamberts et al., 1985). There is a recent review (Lamberts, 1986) of the effects of SMS 201-995 on GH release, and its possible application in the treatment of gastrointestinal disorders, diabetes mellitus and oncology, but so far little is known about its influence on the central nervous system or its behavioral effects.

The data available on the grooming/scratching-inducing effect of somatostatin but not of its analog $\left[\mathrm{D}-\operatorname{Trp}^{8}\right]$ somatostatin made it of interest to investigate whether the somatostatin analog SMS 201-995 would induce excessive grooming and if so, to compare the composition of the grooming thus induced with that of grooming induced by the parent molecule. The present study was designed for this purpose and the excessive grooming inducing effects of SMS 201-995 and somatostatin were further investigated by evaluation of the composition of the grooming behavior elicited by these peptides. The possible suppression of this excessive grooming by haloperidol, naloxone and neurotensin was also investigated. Finally, experiments were performed on the occurrence of tolerance with respect to peptide-induced grooming.

\section{Materials and methods}

Male rats from an inbred Wistar strain (TNO, Zeist, The Netherlands), weighing approximately $150 \mathrm{~g}$ were used. The brain ventricular system was cannulated for i.c.v. injection of the peptides by placing a polyethylene cannula in the right lateral ventricle. This operation was performed under Hypnorm $^{60}$ (5.0 mg fluanison and $0.2 \mathrm{mg}$ fentanyl per $0.5 \mathrm{ml} / \mathrm{kg}$ s.c.; Duphar B.V., Amsterdam, The Netherlands) anesthesia. The animals were allowed to recover from the surgery for 5 days and were single-caged under standard light-dark conditions (light on 07:00 a.m., off 07:00 p.m.) with food and water ad libitum. The rats were allocated randomly to different treatment groups and received i.c.v. injections of $2 \mu \mathrm{l}$. Each animal was used only once. Somatostatin (Aminopan ${ }^{\text {Bs }}$, B 150. UCB, Breda, The Netherlands) was injected in graded doses of $30 \mathrm{pg}, 100 \mathrm{pg}, 300 \mathrm{ng}$ and $1 \mu \mathrm{g}$ ). SMS 201-995 (Sandostatin ${ }^{\sqrt{8}}$ ) was injected i.c.v. in graded doses of 10,50,100, 200 and $300 \mathrm{ng}$. Pretreatment with naloxone (Endo Labs., New York; $1 \mathrm{mg} / \mathrm{kg}$ s.c.) or with haloperidol $(0.05$ $\mathrm{mg} / \mathrm{kg}$ s.c.) was performed at 20 min prior to the i.c.v. injection of somatostatin or SMS 201-995 (Van Wimersma Greidanus et al., 1985b; 1986). Pretreatment with neurotensin (B 560, UCB, Breda. The Netherlands, $1 \mu \mathrm{g}$ i.c.v.) was performed immediately prior to the i.c.v. injection of somatostatin or SMS 201-995. An experiment was performed to find whether systemic administration of SMS 201-995 would induce excessive grooming. Doses of 20 and $900 \mu \mathrm{g}$ of the peptide were injected s.c. for the purpose. Artificial CSF was used as placebo for i.c.v. injection and saline served as placebo for s.c. administration.

Experiments to investigate whether or not tolerance would develop to the grooming-inducing effect of somatostatin and/or SMS 201-995 were designed as in previous studies with other groom- 
ing-inducing hormones (Jolles et al., 1978; Aloyo et al., 1983; Van Wimersma Greidanus et al., 1985a) in which animals were injected i.c.v. with (1) an initial dose (200 ng) of SMS 201-995 (pretreatment) followed $4 \mathrm{~h}$ later by a dose of $50 \mathrm{ng}$ SMS 201-995, or placebo (treatment); or (2) an initial dose of $1 \mu \mathrm{g}$ of somatostatin (pretreatment), followed $4 \mathrm{~h}$ later by a dose of $1 \mu \mathrm{g}$ somatostatin, or placebo (treatment). Groups treated initially with placebo (pretreatment) and $4 \mathrm{~h}$ later with the peptide (200 ng SMS 201-995 or $1 \mu \mathrm{g}$ somatostatin, treatment) were also included. To study whether cross-tolerance between SMS 201-995 and somatostatin would occur, groups of rats were included which received SMS 201-995 (200 ng) as pretreatment followed by somatostatin $(1 \mu \mathrm{g})$ and somatostatin $(1 \mu \mathrm{g})$ as pretreatment, followed by SMS 201-995 (50 ng).

The grooming test was performed according to Gispen et al. (1975). Briefly, the rats were placed into Perspex observation boxes $(26 \times 10 \times 13 \mathrm{~cm})$, immediately after i.c.v. or s.c. injection of somatostatin or SMS 201-995. Scoring of grooming frequencies was started $10 \mathrm{~min}$ later, with a $15 \mathrm{~s}$ sampling procedure for a period of $50 \mathrm{~min}$, resulting in a theoretically maximal total grooming score (T) of 200. The following elements of grooming were scored: head washing $(\mathrm{H})$, body grooming (B), paw licking ( $\mathrm{P})$, sexual (ano-genital) grooming + tail licking (A), scratching (S). Results are expressed as mean ( \pm S.E.M.) grooming score per treatment group for $50 \mathrm{~min}$. The percentages presented in the tables represent the proportion of the total grooming score $(\mathrm{T}=100 \%)$ displayed by the rats following a particular treatment. Statistical evaluation of the data was performed by analysis of variance (ANOVA) in combination with a supplemental t-test (Bruning and Kintz, 1977).

\section{Results}

After its i.c.v. administration, SMS 201-995 induced dose-dependent excessive grooming in the dose range $10-50 \mathrm{ng}$. Higher doses did not further increase the total grooming scores $(\mathrm{T})$, although only approximately $60 \%$ of the theoretical total grooming score $(T=200)$ was usually displayed by animals treated with doses of 100,200 or 300 ng (table 1). However, the composition of the SMS 201-995-induced excessive grooming changed slightly when higher doses were applied. The contribution of the scratching (S) element amounted to $18.4 \%$ in rats treated with $10 \mathrm{ng}$, increasing to $43.6 \%$ following injection of $50 \mathrm{ng}$. Higher doses resulted in a gradual decline in the contribution of this element, accompanied by a slight increase of the body grooming (B) element.

Systemic administration of SMS 201-995 did not induce excessive grooming. Neither total grooming scores $(\mathrm{T})$ nor the scores for any grooming element were significantly increased following s.c. administration of SMS 201-995 in doses up to $900 \mu \mathrm{g}$ (table 1). However, the relative distribution of the grooming elements, as calculated for the grooming displayed by animals treated s.c. with $900 \mu \mathrm{g}$, differed slightly from that of placebotreated rats. A small shift in the composition of grooming towards relatively more scratching (S) was seen in rats that received s.c. the higher dose of SMS 201-995 than in their controls.

Excessive grooming followed relatively high (i.c.v.) doses of somatostatin (table 2). Only $1 \mu \mathrm{g}$ resulted in significantly higher total grooming scores (T), lower doses (30, 100 and $300 \mathrm{ng}$ ) did not induce an increase in total grooming. The increase of total grooming frequencies seen after 1 $\mu \mathrm{g}$ somatostatin was due to a marked increase in the scratching (S) element. Interestingly, animals treated with 100 or $300 \mathrm{ng}$ somatostatin also displayed a slightly significant increase in scratching, which, however, was not sufficient to result in a significantly higher total grooming score.

Naloxone suppressed the excessive grooming induced by $50 \mathrm{ng}$ SMS 201-995, mainly by a reduction of scratching (S) and, to a lesser extent, body grooming (B). Naloxone suppressed the excessive grooming induced by somatostatin similarly (table 3). Novelty-induced excessive grooming also, as displayed by placebo-treated rats, was reduced by pretreatment with naloxone. This reduction was due mainly to a decrease in the scores for body grooming (B) and head washing (H).

Haloperidol suppressed the novelty-induced grooming of placebo-treated rats as well as soma- 
TABLE 1

Grooming scores following i.c.v. and s.c. administration of graded doses of SMS 201-995.

\begin{tabular}{|c|c|c|c|c|c|c|c|}
\hline & $N$ & $\mathrm{H}$ & B & A & $\mathrm{P}$ & $\mathrm{S}$ & $\mathrm{T}$ \\
\hline i.c.v. CSF & 13 & $\begin{array}{l}10.2 \pm 1.3 \\
30.2 \%\end{array}$ & $\begin{array}{l}9.2 \pm 1.4 \\
27.2 \%\end{array}$ & $\begin{array}{l}2.8 \pm 0.7 \\
8.3 \%\end{array}$ & $\begin{array}{l}9.7 \pm 0.8 \\
28.7 \%\end{array}$ & $\begin{array}{l}2.0 \pm 0.8 \\
5.9 \%\end{array}$ & $33.8 \pm 3.8$ \\
\hline $10 \mathrm{ng}$ & 10 & $\begin{array}{l}22.5 \pm 4.3^{a} \\
38.4 \%\end{array}$ & $\begin{array}{l}12.0 \pm 3.6 \\
20.5 \%\end{array}$ & $\begin{array}{l}2.4 \pm 1.0 \\
4.1 \%\end{array}$ & $\begin{array}{l}10.9 \pm 1.2 \\
18.6 \%\end{array}$ & $\begin{array}{l}10.8 \pm 5.7 \\
18.4 \%\end{array}$ & $58.6 \pm 7.8^{a}$ \\
\hline $50 \mathrm{ng}$ & 15 & $\begin{array}{l}35.0 \pm 3.7^{b} \\
29.7 \%\end{array}$ & $\begin{array}{l}10.7 \pm 1.1 \\
9.1 \%\end{array}$ & $\begin{array}{l}2.4 \pm 0.5 \\
2.0 \%\end{array}$ & $\begin{array}{l}18.3 \pm 2.2^{a} \\
15.5 \%\end{array}$ & $\begin{array}{l}51.3 \pm 6.5^{b} \\
43.6 \%\end{array}$ & $117.7 \pm 5.7^{b}$ \\
\hline $100 \mathrm{ng}$ & 16 & $\begin{array}{l}27.4 \pm 4.6^{a} \\
21.9 \%\end{array}$ & $\begin{array}{l}15.6 \pm 3.4 \\
12.5 \%\end{array}$ & $\begin{array}{l}4.4 \pm 1.2 \\
3.5 \%\end{array}$ & $\begin{array}{l}28.3 \pm 4.0 \\
22.6 \%\end{array}$ & $\begin{array}{l}49.4 \pm 9.9^{b} \\
39.5 \%\end{array}$ & $125.1 \pm 14.0^{b}$ \\
\hline $200 \mathrm{ng}$ & 15 & $\begin{array}{l}40.7 \pm 3.6^{b} \\
33.5 \%\end{array}$ & $\begin{array}{l}19.5 \pm 3.2^{\mathrm{a}} \\
16.1 \%\end{array}$ & $\begin{array}{l}4.8 \pm 1.1 \\
4.0 \%\end{array}$ & $\begin{array}{l}19.1 \pm 2.1^{a} \\
15.8 \%\end{array}$ & $\begin{array}{l}37.3 \pm 6.9 \\
30.7 \%\end{array}$ & $121.5 \pm 8.5^{h}$ \\
\hline $300 \mathrm{ng}$ & 13 & $\begin{array}{l}30.8 \pm 3.8^{a} \\
25.4 \%\end{array}$ & $\begin{array}{l}23.3 \pm 4.1^{\mathrm{a}} \\
19.2 \%\end{array}$ & $\begin{array}{l}5.5 \pm 1.8 \\
4.5 \%\end{array}$ & $\begin{array}{l}31.2 \pm 2.5^{b} \\
25.8 \%\end{array}$ & $\begin{array}{l}30.2 \pm 7.3^{a} \\
24.9 \%\end{array}$ & $121.1 \pm 12.0^{b}$ \\
\hline s.c. saline & 6 & $\begin{array}{l}5.8 \pm 0.9 \\
29.0 \%\end{array}$ & $\begin{array}{l}7.5 \pm 1.8 \\
37.5 \%\end{array}$ & $\begin{array}{l}2.0 \pm 1.1 \\
10.0 \%\end{array}$ & $\begin{array}{l}3.5 \pm 1.2 \\
17.5 \%\end{array}$ & $\begin{array}{l}1.2 \pm 0.3 \\
6.0 \%\end{array}$ & $20.0 \pm 3.5$ \\
\hline $20 \mu \mathrm{g}$ & 8 & $\begin{array}{l}8.4 \pm 1.4 \\
37.5 \%\end{array}$ & $\begin{array}{l}6.6 \pm 1.6 \\
29.5 \%\end{array}$ & $\begin{array}{l}1.5 \pm 0.5 \\
6.7 \%\end{array}$ & $\begin{array}{l}4.4 \pm 0.8 \\
19.6 \%\end{array}$ & $\begin{array}{l}1.5 \pm 0.7 \\
6.7 \%\end{array}$ & $22.4 \pm 3.9$ \\
\hline $900 \mu \mathrm{g}$ & 5 & $\begin{array}{l}3.4 \pm 1.4 \\
21.5 \%\end{array}$ & $\begin{array}{l}2.0 \pm 1.0 \\
12.7 \%\end{array}$ & $\begin{array}{l}1.8 \pm 0.7 \\
11.4 \%\end{array}$ & $\begin{array}{l}5.0 \pm 1.6 \\
31.6 \%\end{array}$ & $\begin{array}{l}3.6 \pm 1.4 \\
22.8 \%\end{array}$ & $15.8 \pm 5.1$ \\
\hline
\end{tabular}

${ }^{a} \mathrm{P} \leqslant 0.05$ (vs. CSF); ${ }^{\mathrm{b}} \mathrm{P} \leqslant 0.01$ (vs. CSF).

tostatin-induced excessive grooming (table 4). Although most elements seemed to be involved in this suppressive effect of haloperidol, the reduction of total grooming scores ( $\mathrm{T}$ ) by pretreatment with this drug was due mainly to a reduction of the body grooming (B) and paw licking (P) elements in placebo-treated rats and to a reduction in body grooming $(\mathrm{B})$, paw licking $(\mathrm{P})$ and scratching $(S)$ in somatostatin-treated rats. In contrast, haloperidol did not reduce the total grooming scores (T) in SMS 201-995-treated animals, al- though the scores for head washing $(\mathrm{H})$ and paw licking $(\mathrm{P})$ were significantly reduced in animals treated with haloperidol and SMS 201-995, as compared with rats injected only with SMS 201995. However, these reductions were accompanied by a marked increase in scratching (S), resulting in similar total grooming scores for both groups of animals. Haloperidol pretreatment thus resulted in a marked shift in the relative distribution of grooming elements in SMS 201-995-treated rats, which was not observed in placebo-treated rats,

\section{TABLE 2}

Grooming scores following i.c.v. administration of graded doses of somatostatin.

\begin{tabular}{|c|c|c|c|c|c|c|c|}
\hline & $\mathrm{N}$ & $\mathrm{H}$ & B & A & $\mathrm{P}$ & $S$ & $\mathrm{~T}$ \\
\hline $\mathrm{CSF}$ & 9 & $\begin{array}{l}7.9 \pm 1.0 \\
21.0 \%\end{array}$ & $\begin{array}{l}14.3 \pm 2.4 \\
37.9 \%\end{array}$ & $\begin{array}{l}2.7 \pm 0.8 \\
7.2 \%\end{array}$ & $\begin{array}{l}12.0 \pm 2.0 \\
31.8 \%\end{array}$ & $\begin{array}{l}0.8 \pm 0.4 \\
2.1 \%\end{array}$ & $37.7 \pm 3.5$ \\
\hline $30 \mathrm{ng}$ & 7 & $\begin{array}{l}7.3 \pm 1.5 \\
27.4 \%\end{array}$ & $\begin{array}{l}6.7 \pm 2.3^{a} \\
25.2 \%\end{array}$ & $\begin{array}{l}2.1 \pm 1.0 \\
7.9 \%\end{array}$ & $\begin{array}{l}8.0 \pm 2.0 \\
30.1 \%\end{array}$ & $\begin{array}{l}2.4 \pm 2.1 \\
9.0 \%\end{array}$ & $26.6 \pm 5.1$ \\
\hline $100 \mathrm{ng}$ & 7 & $\begin{array}{l}10.1 \pm 1.2 \\
23.5 \%\end{array}$ & $\begin{array}{l}9.9 \pm 2.0 \\
23.1 \%\end{array}$ & $\begin{array}{l}5.9 \pm 1.8 \\
13.8 \%\end{array}$ & $\begin{array}{l}11.0 \pm 2.8 \\
25.6 \%\end{array}$ & $\begin{array}{l}6.0 \pm 2.6^{a} \\
14.0 \%\end{array}$ & $42.9 \pm 7.7$ \\
\hline $300 \mathrm{ng}$ & 8 & $\begin{array}{l}6.6 \pm 0.9 \\
14.8 \%\end{array}$ & $\begin{array}{l}13.8 \pm 1.6 \\
31.0 \%\end{array}$ & $\begin{array}{l}2.4 \pm 0.8 \\
5.4 \%\end{array}$ & $\begin{array}{l}8.0 \pm 0.9 \\
18.0 \%\end{array}$ & $\begin{array}{l}13.8 \pm 3.6^{a} \\
31.0 \%\end{array}$ & $44.5 \pm 4.8$ \\
\hline $1 \mu \mathrm{g}$ & 8 & $\begin{array}{l}6.1 \pm 1.4 \\
9.6 \%\end{array}$ & $\begin{array}{l}10.4 \pm 2.5 \\
16.4 \%\end{array}$ & $\begin{array}{l}3.1 \pm 0.7 \\
4.9 \%\end{array}$ & $\begin{array}{l}13.8 \pm 4.4 \\
21.7 \%\end{array}$ & $\begin{array}{l}30.1 \pm 9.5^{a} \\
47.4 \%\end{array}$ & $63.5 \pm 9.7^{\mathrm{a}}$ \\
\hline
\end{tabular}

${ }^{a} \mathrm{P} \leqslant 0.05$ vs. CSF. 
TABLE 3

Influence of naloxone (NAL; $1 \mathrm{mg} / \mathrm{kg}$; s.c.; $\mathrm{t}=-20 \mathrm{~min}$ ) on excessive grooming induced by i.c.v. administration of SMS $201-995$ (50 $\mathrm{ng} ; \mathrm{t}=0)$ or somatostatin $(1 \mu \mathrm{g} ; \mathrm{t}=0)$.

\begin{tabular}{|c|c|c|c|c|c|c|c|}
\hline & $\mathbf{N}$ & $\mathrm{H}$ & B & A & $\mathrm{P}$ & $\mathrm{S}$ & $T$ \\
\hline $\mathrm{CSF}$ & 6 & $\begin{array}{l}13.0 \pm 3.1 \\
39.8 \%\end{array}$ & $\begin{array}{l}10.5 \pm 1.6 \\
32.1 \%\end{array}$ & $\begin{array}{l}2.7 \pm 1.3 \\
8.3 \%\end{array}$ & $\begin{array}{l}6.9 \pm 0.9 \\
18.3 \%\end{array}$ & $\begin{array}{l}0.5 \pm 0.5 \\
1.5 \%\end{array}$ & $32.7 \pm 4.9$ \\
\hline NAL & 6 & $\begin{array}{l}3.0 \pm 0.7^{\mathrm{a}} \\
22.6 \%\end{array}$ & $\begin{array}{l}2.3 \pm 1.2^{\mathrm{a}} \\
17.3 \%\end{array}$ & $\begin{array}{l}1.8 \pm 0.9 \\
13.5 \%\end{array}$ & $\begin{array}{l}5.7 \pm 2.4 \\
42.9 \%\end{array}$ & $\begin{array}{l}0.5 \pm 0.3 \\
3.8 \%\end{array}$ & $13.3 \pm 4.5^{\mathrm{a}}$ \\
\hline SMS & 15 & $\begin{array}{l}43.3 \pm 6.5^{a} \\
43.3 \%\end{array}$ & $\begin{array}{l}10.8 \pm 2.5 \\
10.8 \%\end{array}$ & $\begin{array}{l}1.5 \pm 0.5 \\
1.5 \%\end{array}$ & $\begin{array}{l}7.3 \pm 1.6 \\
7.3 \%\end{array}$ & $\begin{array}{l}37.1 \pm 6.6^{b} \\
37.1 \%\end{array}$ & $99.9 \pm 12.1^{\mathrm{a}}$ \\
\hline $\mathrm{SMS}+\mathrm{NAL}$ & 14 & $\begin{array}{l}27.4 \pm 5.8^{a} \\
56.1 \%\end{array}$ & $\begin{array}{l}4.3 \pm 1.4^{\mathrm{a}, \mathrm{c}} \\
8.8 \%\end{array}$ & $\begin{array}{l}1.0 \pm 0.3 \\
2.0 \%\end{array}$ & $\begin{array}{l}5.6 \pm 0.8 \\
11.5 \%\end{array}$ & $\begin{array}{l}10.5 \pm 3.2^{\mathrm{acc}} \\
21.5 \%\end{array}$ & $48.8 \pm 8.1^{c}$ \\
\hline Somatostatin & 6 & $\begin{array}{l}13.3 \pm 1.0 \\
19.1 \%\end{array}$ & $\begin{array}{l}18.8 \pm 1.3^{a} \\
26.9 \%\end{array}$ & $\begin{array}{l}5.7 \pm 0.8 \\
8.2 \%\end{array}$ & $\begin{array}{l}13.5 \pm 1.6^{a} \\
19.3 \%\end{array}$ & $\begin{array}{l}18.5 \pm 5.4^{a} \\
26.5 \%\end{array}$ & $69.8 \pm 4.3^{a}$ \\
\hline $\begin{array}{l}\text { Somatostatin } \\
\text { + NAL }\end{array}$ & 7 & $\begin{array}{l}8.0 \pm 2.4 \\
23.1 \%\end{array}$ & $\begin{array}{l}8.4 \pm 2.3^{d} \\
24.3 \%\end{array}$ & $\begin{array}{l}3.9 \pm 1.8 \\
11.3 \%\end{array}$ & $\begin{array}{l}9.6 \pm 2.5 \\
27.7 \%\end{array}$ & $\begin{array}{l}4.7 \pm 2.2^{d} \\
13.6 \%\end{array}$ & $34.6 \pm 6.2^{d}$ \\
\hline
\end{tabular}

${ }^{a} \mathrm{P} \leqslant 0.05$ (vs. CSF); ${ }^{\mathrm{b}} \mathrm{P} \leqslant 0.01$ (vs. CSF); ${ }^{\mathrm{c}} \mathrm{P} \leqslant 0.05$ (vs. SMS); ${ }^{\mathrm{d}} \mathrm{P} \leqslant 0.05$ (vs. somatostatin).

nor in somatostatin-treated animals.

Neurotensin markedly suppressed the noveltyinduced grooming of the placebo-treated rats and the excessive grooming induced by somatostatin (table 5). This suppressive effect of neurotensin involved almost all elements of grooming in both cases. However, neurotensin did not suppress the excessive grooming induced by SMS 201-995. Neither total grooming scores $(T)$ nor any score of the various elements displayed by the rats treated with neurotensin/SMS 201-995 was significantly different from those observed in animals treated with placebo/SMS 201-995 (table 5). An additional experiment was performed with $3 \mu \mathrm{g}$ neurotensin, and this higher dose of neurotensin had no effect on SMS 201-995-induced grooming but novelty-induced grooming was markedly reduced (data not shown).

In the course of the experiments on the development of tolerance to the grooming inducing effects of somatostatin and SMS 201-995 it appeared that i.c.v. administration of somatostatin doses higher than $1 \mu \mathrm{g}$ induced barrel rotation and frequently resulted in death of the animals. Since

TABLE 4

Influence of haloperidol (HP; $0.05 \mathrm{mg} / \mathrm{kg}$; s.c. $\mathrm{t}=-20 \mathrm{~min}$ ) on excessive grooming induced by i.c.v. administration of SMS $201-995$ (50 $\mathrm{ng} ; \mathrm{t}=0$ ) or somatostatin $(1 \mu \mathrm{g} ; \mathrm{t}=0)$.

\begin{tabular}{|c|c|c|c|c|c|c|c|}
\hline & $\mathrm{N}$ & $\mathrm{H}$ & B & $\mathrm{A}$ & $\mathbf{P}$ & $S$ & $\mathrm{~T}$ \\
\hline$\overline{\mathrm{CSF}}$ & 7 & $\begin{array}{l}8.2 \pm 2.1 \\
20.5 \%\end{array}$ & $\begin{array}{l}16.9 \pm 1.4 \\
42.3 \%\end{array}$ & $\begin{array}{l}3.7 \pm 0.9 \\
9.3 \%\end{array}$ & $\begin{array}{l}9.1 \pm 1.8 \\
22.8 \%\end{array}$ & $\begin{array}{l}2.0 \pm 0.7 \\
5.0 \%\end{array}$ & $40.0 \pm 2.7$ \\
\hline HP & 7 & $\begin{array}{l}3.9 \pm 0.8 \\
27.9 \%\end{array}$ & $\begin{array}{l}4.1 \pm 1.5^{a} \\
29.3 \%\end{array}$ & $\begin{array}{l}1.7 \pm 0.7 \\
12.1 \%\end{array}$ & $\begin{array}{l}3.7 \pm 1.6^{a} \\
26.4 \%\end{array}$ & $\begin{array}{l}0.6 \pm 0.4 \\
4.3 \%\end{array}$ & $14.0 \pm 4.3^{\mathrm{a}}$ \\
\hline SMS & 9 & $\begin{array}{l}45.1 \pm 4.9^{b} \\
37.7 \%\end{array}$ & $\begin{array}{l}8.3 \pm 1.6 \\
6.9 \%\end{array}$ & $\begin{array}{l}2.7 \pm 1.6 \\
2.3 \%\end{array}$ & $\begin{array}{l}8.1 \pm 1.2 \\
6.8 \%\end{array}$ & $\begin{array}{l}55.3 \pm 7.0^{b} \\
46.2 \%\end{array}$ & $119.6 \pm 7.0^{b}$ \\
\hline SMS + HP & 8 & $\begin{array}{l}22.1 \pm 3.6^{c} \\
18.3 \%\end{array}$ & $\begin{array}{l}4.9 \pm 0.9 \\
4.1 \%\end{array}$ & $\begin{array}{l}1.3 \pm 0.6 \\
1.1 \%\end{array}$ & $\begin{array}{l}4.3 \pm 1.2^{c} \\
3.6 \%\end{array}$ & $\begin{array}{l}88.1 \pm 8.2^{\circ} \\
73.1 \%\end{array}$ & $120.6 \pm 7.0$ \\
\hline Somatostatin & 6 & $\begin{array}{l}11.0 \pm 3.4 \\
12.2 \%\end{array}$ & $\begin{array}{l}26.2 \pm 1.8^{a} \\
29.2 \%\end{array}$ & $\begin{array}{l}5.2 \pm 1.8 \\
5.8 \%\end{array}$ & $\begin{array}{l}20.2 \pm 5.7^{a} \\
22.5 \%\end{array}$ & $\begin{array}{l}27.3 \pm 6.5^{a} \\
30.4 \%\end{array}$ & $89.8 \pm 6.5^{a}$ \\
\hline $\begin{array}{l}\text { Somatostatin } \\
+\mathrm{HP}\end{array}$ & 8 & $\begin{array}{l}7.6 \pm 2.7 \\
21.2 \%\end{array}$ & $\begin{array}{l}10.0 \pm 2.8^{d} \\
27.9 \%\end{array}$ & $\begin{array}{l}3.3 \pm 0.7 \\
9.2 \%\end{array}$ & $\begin{array}{l}7.9 \pm 1.8^{\mathrm{d}} \\
22.0 \%\end{array}$ & $\begin{array}{l}7.1 \pm 2.7^{d} \\
19.8 \%\end{array}$ & $35.9 \pm 7.4^{d}$ \\
\hline
\end{tabular}

${ }^{\mathrm{a}} \mathrm{P} \leqslant 0.05$ (vs. CSF); ${ }^{\mathrm{b}} \mathrm{P} \leqslant 0.01$ (vs. CSF); ${ }^{\mathrm{c}} \mathrm{P} \leqslant 0.05$ (vs. SMS); ${ }^{\mathrm{d}} \mathrm{P} \leqslant 0.01$ (vs. somatostatin). 
TABLE 5

Influence of neurotensin (NT; $1 \mu \mathrm{g}$; i.c.v.: $\mathrm{t}=0$ ) on excessive grooming induced by SMS 201-995 (50 ng; i.c.v.; $\mathrm{t}=0)$ or somatostatin $(1 \mu$ g; i.c.v.; $\mathrm{t}=0)$.

\begin{tabular}{|c|c|c|c|c|c|c|c|}
\hline & $\mathrm{N}$ & $\mathbf{H}$ & $\mathrm{B}$ & A & $\mathrm{P}$ & $\mathrm{S}$ & $\mathrm{T}$ \\
\hline$\overline{\mathrm{CSF}}$ & 6 & $\begin{array}{l}6.2 \pm 0.9 \\
17.5 \%\end{array}$ & $\begin{array}{l}10.8 \pm 1.8 \\
30.4 \%\end{array}$ & $\begin{array}{l}5.8 \pm 2.3 \\
16.3 \%\end{array}$ & $\begin{array}{l}8.7 \pm 2.2 \\
24.5 \%\end{array}$ & $\begin{array}{r}4.0 \pm 1.6 \\
11.3 \%\end{array}$ & $35.5 \pm 5.6$ \\
\hline NT & 6 & $\begin{array}{l}3.2 \pm 0.9 \\
21.8 \%\end{array}$ & $\begin{array}{l}6.3 \pm 1.1^{a} \\
42.9 \%\end{array}$ & $\begin{array}{l}1.2 \pm 0.7^{a} \\
8.2 \%\end{array}$ & $\begin{array}{l}4.0 \pm 0.6^{a} \\
27.2 \%\end{array}$ & $\begin{array}{l}0 \\
0\end{array}$ & $14.7 \pm 2.6^{a}$ \\
\hline SMS & 6 & $\begin{array}{l}12.5 \pm 2.2^{a} \\
8.3 \%\end{array}$ & $\begin{array}{l}12.5 \pm 3.2 \\
8.3 \%\end{array}$ & $\begin{array}{l}0.7 \pm 0.3^{a} \\
0.5 \%\end{array}$ & $\begin{array}{l}27.2 \pm 7.5^{a} \\
18.0 \%\end{array}$ & $\begin{array}{l}98.5 \pm 9.4^{b} \\
65.1 \%\end{array}$ & $151.3 \pm 9.9^{b}$ \\
\hline $\mathrm{SMS}+\mathrm{NT}$ & 5 & $\begin{array}{l}18.2 \pm 3.9^{a} \\
13.0 \%\end{array}$ & $\begin{array}{l}9.0 \pm 2.3 \\
6.4 \%\end{array}$ & $\begin{array}{l}1.4 \pm 0.6 \\
1.0 \%\end{array}$ & $\begin{array}{l}22.6 \pm 2.6^{a} \\
16.1 \%\end{array}$ & $\begin{array}{l}89.2 \pm 17.5^{b} \\
63.5 \%\end{array}$ & $140.4 \pm 13.9^{h}$ \\
\hline Somatostatin & 6 & $\begin{array}{l}13.3 \pm 2.3^{a} \\
16.9 \%\end{array}$ & $\begin{array}{l}18.7 \pm 3.5 \\
23.8 \%\end{array}$ & $\begin{array}{l}3.0 \pm 0.8 \\
3.8 \%\end{array}$ & $\begin{array}{l}20.6 \pm 3.7^{a} \\
25.4 \%\end{array}$ & $\begin{array}{l}23.7 \pm 5.8^{a} \\
30.1 \%\end{array}$ & $78.7 \pm 6.8^{a}$ \\
\hline $\begin{array}{l}\text { Somatostatin } \\
+\mathrm{NT}\end{array}$ & 8 & $\begin{array}{l}4.9 \pm 0.8^{\mathrm{c}} \\
23.6 \%\end{array}$ & $\begin{array}{l}7.3 \pm 2.0^{c} \\
35.1 \%\end{array}$ & $\begin{array}{l}1.8 \pm 0.6 \\
8.7 \%\end{array}$ & $\begin{array}{l}4.4 \pm 0.8^{c} \\
21.2\end{array}$ & $\begin{array}{r}2.5 \pm 1.3^{c} \\
12.0 \%\end{array}$ & $20.8 \pm 4.4^{\mathrm{c}}$ \\
\hline
\end{tabular}

${ }^{a} \mathrm{P} \leqslant 0.05$ (vs. CSF); ${ }^{b} \mathrm{P} \leqslant 0.01$ (vs. CSF); ${ }^{c} \mathrm{P} \leqslant 0.05$ (vs. somatostatin).

doses lower than $1 \mu \mathrm{g}$ did not result in a significant increase of total grooming scores (see before), it was decided to use a $1 \mu \mathrm{g}$ dose of this peptide for the initial injection (pretreatment) and the same dose for the second injection (treatment). The administration of $50 \mathrm{ng}$ SMS 201-995 (table 6) did not induce excessive grooming in animals pretreated with $200 \mathrm{ng}$ of this peptide. Likewise 1

TABLE 6

Induction of various elements of grooming by repeated i.c.v. injection of SMS 201-995 and/or somatostatin

\begin{tabular}{|c|c|c|c|c|c|c|c|c|}
\hline & & $\mathrm{N}$ & $\mathrm{H}$ & B & A & $\mathrm{P}$ & $\mathrm{S}$ & $T$ \\
\hline Pretreatment & $\mathrm{CSF}$ & 8 & $11.3 \pm 0.9$ & $9.9 \pm 2.5$ & $2.4 \pm 1.3$ & $6.3 \pm 0.9$ & $0.4 \pm 0.3$ & $30.1 \pm 3.6$ \\
\hline Treatment & CSF & 8 & $7.5 \pm 0.9$ & $9.5 \pm 1.6$ & $3.3 \pm 1.1$ & $5.0 \pm 1.5$ & $1.0 \pm 0.3$ & $26.3 \pm 3.8$ \\
\hline Pretreatment & $\mathrm{CSF}$ & 12 & $7.1 \pm 1.4$ & $7.8 \pm 1.7$ & $2.6 \pm 0.9$ & $7.7 \pm 2.2$ & $4.5 \pm 1.8$ & $9.7 \pm 5.7$ \\
\hline Treatment & SMS $50 \mathrm{ng}$ & 12 & $34.4 \pm 5.3$ & $10.6 \pm 1.9$ & $2.1 \pm 0.5$ & $11.3 \pm 1.5$ & $52.8 \pm 6.8$ & $111.3 \pm 10.8$ \\
\hline Pretreatment & SMS $200 \mathrm{ng}$ & 11 & $44.5 \pm 6.1$ & $21.8 \pm 4.3$ & $6.8 \pm 1.6$ & $18.8 \pm 2.0$ & $27.6 \pm 4.9$ & $119.6 \pm 6.7$ \\
\hline Treatment & SMS $50 \mathrm{ng}$ & 11 & $5.0 \pm 1.5^{a}$ & $6.4 \pm 1.5$ & $2.9 \pm 1.1$ & $6.4 \pm 1.9$ & $10.7 \pm 5.4^{a}$ & $31.4 \pm 9.2^{a}$ \\
\hline Pretreatment & SMS $200 \mathrm{ng}$ & 7 & $45.7 \pm 3.6$ & $30.0 \pm 3.2$ & $8.7 \pm 1.9$ & $15.6 \pm 2.5$ & $26.3 \pm 6.4$ & $126.3 \pm 9.3$ \\
\hline Treatment & $\mathrm{CSF}$ & 7 & $3.1 \pm 0.6$ & $5.6 \pm 2.5$ & $2.1 \pm 0.7$ & $4.9 \pm 1.4$ & $5.7 \pm 2.5$ & $21.4 \pm 6.1$ \\
\hline Pretreatment & CSF & 11 & $4.9 \pm 0.8$ & $11.5 \pm 1.3$ & $2.2 \pm 0.5$ & $6.2 \pm 0.9$ & $1.5 \pm 0.4$ & $26.2 \pm 2.3$ \\
\hline Treatment & Somatostatin $1 \mu \mathrm{g}$ & 11 & $6.1 \pm 1.0$ & $10.0 \pm 1.9$ & $2.5 \pm 0.6$ & $9.1 \pm 1.9$ & $17.8 \pm 2.8$ & $45.5 \pm 4.8$ \\
\hline Pretreatment & Somatostatin $1 \mu \mathrm{g}$ & 16 & $8.5 \pm 1.0$ & $15.0 \pm 2.5$ & $3.6 \pm 0.9$ & $9.0 \pm 1.5$ & $11.8 \pm 3.4$ & $47.8 \pm 7.2$ \\
\hline Treatment & Somatostatin $1 \mu \mathrm{g}$ & 16 & $3.1 \pm 0.7^{b}$ & $3.0 \pm 0.8^{b}$ & $0.8 \pm 0.3^{b}$ & $3.3 \pm 1.1^{b}$ & $2.6 \pm 0.9^{b}$ & $12.9 \pm 2.7^{b}$ \\
\hline Pretreatment & Somatostatin $1 \mu \mathrm{g}$ & 11 & $9.4 \pm 2.6$ & $15.0 \pm 3.6$ & $1.6 \pm 0.3$ & $9.0 \pm 1.5$ & $21.3 \pm 4.4$ & $56.3 \pm 9.6$ \\
\hline Treatment & CSF & 11 & $2.0 \pm 0.5$ & $3.2 \pm 1.1$ & $1.1 \pm 0.4$ & $3.3 \pm 0.8$ & $1.3 \pm 1.1$ & $10.8 \pm 2.9$ \\
\hline Pretreatment & SMS $200 \mathrm{ng}$ & 11 & $29.6 \pm 6.2$ & $17.1 \pm 2.9$ & $4.5 \pm 1.5$ & $21.8 \pm 2.9$ & $52.0 \pm 9.2$ & $125.0 \pm 9.0$ \\
\hline Treatment & Somatostatin $1 \mu \mathrm{g}$ & 11 & $3.9 \pm 0.8$ & $7.3 \pm 1.6$ & $3.3 \pm 0.9$ & $6.1 \pm 1.0$ & $8.8 \pm 3.4^{b}$ & $29.4 \pm 6.1^{b}$ \\
\hline Pretreatment & Somatostatin $1 \mu \mathrm{g}$ & 10 & $9.1 \pm 1.4$ & $10.8 \pm 2.0$ & $4.1 \pm 1.1$ & $11.9 \pm 3.3$ & $13.9 \pm 3.0$ & $49.8 \pm 7.1$ \\
\hline Treatment & SMS $50 \mathrm{ng}$ & 10 & $9.6 \pm 3.5^{\text {a.c }}$ & $5.0 \pm 1.5^{\mathrm{a}}$ & $2.2 \pm 0.9$ & $8.5 \pm 2.4^{c}$ & $26.5 \pm 12.1^{\mathrm{a}, \mathrm{c}}$ & $51.8 \pm 18.4^{\mathrm{a}, \mathrm{c}}$ \\
\hline
\end{tabular}

${ }^{\mathrm{a}} \mathrm{P} \leqslant 0.05$ (vs. SMS treatment $/ \mathrm{CSF}$ pretreatment); ${ }^{\mathrm{b}} \mathrm{P} \leqslant 0.05$ (vs. somatostatin treatment $/ \mathrm{CSF}$ pretreatment); ${ }^{\mathrm{c}} \mathrm{P} \leqslant 0.05$ (vs. CSF treatment/corresponding pretreatment). 
$\mu \mathrm{g}$ somatostatin did not elicit excessive grooming in rats pretreated $4 \mathrm{~h}$ before with $1 \mu \mathrm{g}$ somatostatin, nor in animals pretreated with $200 \mathrm{ng}$ SMS 201-995. However, 50 ng SMS 201-995 seemed to result in a slight increase in grooming scores in animals pretreated with $1 \mu \mathrm{g}$ somatostatin. Comparison of the grooming score for the latter rats with the score for animals of the somatostatin/CSF group reveals that the total grooming scores (T) displayed by rats of the somatostatin/ SMS group were significantly higher, due mainly to more head washing $(\mathrm{H})$, paw licking $(\mathrm{P})$ and scratching (S).

\section{Discussion}

SMS 201-995 or somatostatin given i.c.v. induced excessive grooming in rats. The groominginducing effect of somatostatin was rather weak and the barrel rotation that followed higher doses confirms findings by others (Cohn and Cohn, 1975). SMS 201-995 did not induce barrel rotation at the doses used, but it appears to be a relatively potent peptide for inducing excessive grooming behavior since an i.c.v. dose as low as $10 \mathrm{ng}$ elicited this behavior. However, doses of $100 \mathrm{ng}$ and more did not further increase the total grooming scores reached with a $50 \mathrm{ng}$ dose of the peptide. These scores were generally about $60 \%$ of the theoretical maximal total grooming scores achievable under the experimental conditions used. This is in contrast with the excessive grooming induced by bombesin and ACTH-(1-24). These peptides elicit excessive grooming in a dose-dependent way and induce total grooming scores which are higher (Van Wimersma Greidanus et al., 1985a,b; 1986) than can be reached with SMS 201-995.

Systemic administration of SMS 201-995 in doses up to $900 \mu \mathrm{g}$ did not result in excessive grooming behavior, while it has been shown (Lamberts et al., 1987) that a single s.c. injection of $10 \mu \mathrm{g} / \mathrm{kg}$ of SMS 201-995 resulted in a significant inhibition of plasma GH levels after $30 \mathrm{~min}$. One of the possible explanations for this phenomenon may be the presence of a very strong blood-brain barrier for this somatostatin analog.
The pattern of the excessive grooming induced by SMS 201-995 and somatostatin is characterized by a predominant display of excessive scratching. Since peptide-induced scratching is mainly due to activation of opiate receptor systems (Van Wimersma Greidanus et al., 1985b; 1986; 1987; Spruijt et al., 1986a) this observation indicates that opiate receptors are involved in the behavioral response to SMS 201-995 and somatostatin. This is consistent with the suggestion by Terenius (1976) that somatostatin acts as partial agonistantagonist on opiate receptors in the CNS, and with other reports on opiate-like actions of somatostatin and some of its analogs (Rezek et al., 1978; Pelton et al., 1985). Interestingly, the contribution of the scratching element to SMS 201995-induced grooming was most pronounced after doses ranging from 50 to $100 \mathrm{ng}$. Administration of higher doses resulted in a grooming behavior in which scratching became less pronounced and other elements were more frequent. This finding could be explained by the assumption that SMS 201-995 acts as a partial agonist-antagonist on opiate receptors as suggested for somatostatin (Terenius, 1975). The observation that naloxone further suppresses the excessive grooming induced by SMS 201-995 and by somatostatin supports the view that opiate receptors are involved in the induced behavior, although the present data do not allow a definite conclusion about a direct interaction of the peptides with these receptors. It was hypothesized that, in this respect, opiate as well as dopaminergic systems were not involved directly in ACTH-induced grooming, but indirectly by the neural circuitry that underlies grooming (Spruijt and Gispen, 1986).

In addition to the difference in grooming inducing potency and dose-effect relationship of the two peptides, there was a marked difference in the effects of haloperidol and neurotensin on the excessive grooming induced by either somatostatin or SMS 201-995. Whereas haloperidol and neurotensin markedly suppress the excessive grooming induced by somatostatin, more or less similarly to the suppression observed for bombesin- and ACTH-induced excessive grooming (Van Wimersma Greidanus et al., 1985b; 1986), no suppressive effect of these substances was seen on SMS 201- 
995-induced total grooming scores. The finding that haloperidol pretreatment of SMS 201-995treated rats caused a shift in the relative distribution of the various grooming elements towards more scratching (S) with a concomittant decrease in body grooming (B) and paw licking (P) could have been due to a blockade of dopamine receptor systems. These receptors are supposed to be generally involved in excessive grooming behavior, resulting in a more predominant and preferential interaction of SMS 201-995 with opiate receptor systems, primarily involved in the scratching response. However, while the present data support that suggestion, further studies are needed to reach more definite conclusions on this issue.

It appears from the results of the experiments on the development of tolerance that, under the experimental conditions used, tolerance occurred to the grooming-inducing effects of SMS 201-995 and of somatostatin. In addition, there was crosstolerance between somatostatin and SMS 201-995. Somatostatin did not induce excessive grooming in rats previously treated with SMS 201-995. However, SMS 201-995 still induced excessive grooming in rats treated with somatostatin $4 \mathrm{~h}$ before, indicating that the cross-tolerance induced by somatostatin for SMS 201-995 was only partial. It is possible that this was due to the marked difference in potency to induce grooming between somatostatin and SMS 201-995.

\section{Acknowledgement}

The authors are grateful to Sandoz for the supply of Sandostatin ${ }^{\mathbb{B}}$.

\section{References}

Aloyo, V.J., B.M. Spruijt, H. Zwiers and W.H. Gispen, 1983 , Peptide-induced excessive grooming: The role of opiate receptors, Peptides 4,833 .

Bauer, W., U. Briner, W. Doepfner, R. Haller, R. Huguenin, P. Marbach, T.J. Petcher and J. Pless, 1982, SMS 201-995: A very potent and selective octapeptide analogue of somatostatin with prolonged action, Life Sci. 31, 1133.

Bruning, J.L. and B.L. Kintz, 1977, Computational Handbook of Statistics. Glenview III, Scott Foresman.

Burke, R.E. and S. Fahn, 1983, Studies of somatostatin-induced barrel rotation in rats, Regul. Pept. 7, 207.
Cohn. M.L. and M. Cohn, 1975, 'Barrel rotation' induced by somatostatin in the non-lesioned rat, Brain Res. 96, 138.

Cools, A.R., V.M. Wiegant and W.H. Gispen, 1978, Distinct dopaminergic systems in ACTH-induced grooming, European J. Pharmacol. 265.

Dobry, J.K.P., M.F. Piercey and L.A. Schroeder, 1981. Pharmacological characterization of scratching behaviour induced by intracranial injection of substance $\mathbf{P}$ and somatostatin, Neuropharmacology 20, 267.

Dodd, J. and J.S. Kelly, 1978, Is somatostatin an excitatory transmitter in the hippocampus?, Nature 273, 674.

Dokas, L.A., H. Zwiers, D.H. Coy and W.H. Gispen, 1983, Somatostatin and analogs inhibit endogenous synaptic plasma membrane protein phosphorylation in vitro, European J. Pharmacol. 88, 185.

Garcia-Sevilla, J.A., T. Magnusson and A. Carlsson, 1978, Effect of intracerebroventricularly administered somatostatin on brain monoamine turnover, Brain Res. 155, 159.

Gispen, W.H. and R.L. Isaacson, 1981, ACTH-induced excessive grooming in the rat, Pharmacol. Ther. 12, 209.

Gispen, W.H., V.M. Wiegant, H. Greven and D. De Wied, 1975, The introduction of excessive grooming in the rat by intraventricular application of peptides derived from ACTH: Structure-activity studies, Life Sci. 17, 645.

Havlicek, V., M. Rezek and H. Friesen, 1976, Somatostatin and thyrotropin releasing hormone: central effect on sleep and motor system, Pharmacol. Biochem. Behav. 4, 455.

Jolles, J., V.M. Wiegant and W.H. Gispen, 1978, Reduced behavioral effectiveness of $\mathrm{ACTH}_{1-24}$ after a second administration: interaction with opiates, Neurosci. Lett. 9, 261.

Lamberts, S.W.J., 1986, Non-pituitary actions of somatostatin. A review on the therapeutic role of SMS 201-995 (sandostatin), Acta Endocrinol. (Suppl.) 276, 41.

Lamberts, S.W.J., R. Oosterom, M. Neufeld and E. Del Pozo, 1985. The somatostatin analog SMS 201-995 induces longacting inhibition of growth hormone secretion without rebound hypersecretion in acromegalic patients, J. Clin. Endocrinol. Metab. 60, 1161.

Lamberts, S.W.J., T. Verleun, J.M. Zuiderwijk and R. Oosterom, 1987, The effect of the somatostatin analog SMS 201-995 on normal growth hormone secretion in the rat, Acta Endocrinol. 115, 196.

Pelton, J.T., K. Gulya, V.J. Hruby, S.P. Duckles and H.I. Yamamura, 1985, Conformationally restricted analogs of somatostatin with high $\mu$-opiate receptor specificity, Proc. Natl. Acad. Sci. U.S.A. 82, 236.

Plotnikoff, N.P., A.J. Kastin and A.V. Schally, 1974, Growth hormone release inhibiting hormone: Neuropharmacological studies, Pharmacol. Biochem. Behav. 2, 693.

Renaud, L.P., J.B. Martin and P. Brazeau, 1975, Depressant action of TRH, LH-RH and somatostatin on activity of central neurones, Nature 255, 233.

Rezek, M., V. Havlicek, L. Leybin, F.S. LaBella and H. Friesen, 1977, Opiate-like naloxone-reversible actions of somatostatin given intracerebrally, Can. J. Physiol. Pharmacol. 56,227

Seybold. V.S., J.L.K. Hylden and G.L. Wilcox, 1982, Intrathe- 
cal substance $P$ and somatostatin in rats: Behaviors indicative of sensation, Peptides 3, 49.

Spruijt, B.M., A.R. Cools, B.A. Ellenbroek and W.H. Gispen, 1986a, Dopaminergic modulation of ACTH-induced grooming, European J. Pharmacol. 120, 249.

Spruijt, B.M., A.R. Cools and W.H. Gispen, 1986b, The periaqueductal gray: a prerequisite for ACTH-induced excessive grooming, Behav. Brain Res. 20, 19.

Spruijt, B.M. and W.H. Gispen, 1983, ACTH and grooming behaviour in the rat, in: Hormones and Vertebrates, eds., J. Balthazar, E. Prove and R. Gilles (Springer-Verlag, Berlin) p. 118.

Spruijt, B.M. and W.H. Gispen, 1986, ACTH and grooming, in: Central Actions of ACTH and Related Peptides, eds. D. De Wied and W. Ferrari (Liviana Press, Padova) p. 179.

Terenius, L., 1976, Somatostatin and ACTH are peptides with partial antagonist-like selectivity for opiate receptors, European J. Pharmacol. 38, 211.

Van Wimersma Greidanus, Tj.B., D.K. Donker, F.F.M. Van Zinnicq Bergmann, R. Bekenkamp, C. Maigret and B. Spruijt, 1985a, Comparison between excessive grooming induced by bombesin or by ACTH: The differential elements of grooming and development of tolerance, Peptides 6,369 .
Van Wimersma Greidanus, Tj.B., D.K. Donker, R. Walhof, J.C.A. Van Grafhorst, N. De Vries, S.J. Van Schaik, C. Maigret, B.M. Spruijt and D.L. Colbern, 1985b, The effects of neurotensin, naloxone and haloperidol on elements of excessive grooming behavior induced by bombesin, Peptides $6,1179$.

Van Wimersma Greidanus, Tj.B., C. Maigret, J.A. Ten Haaf, B.M. Spruijt and D.L. Colbern, 1986, The influence of neurotensin, naloxone, and haloperidol on elements of excessive grooming behavior induced by ACTH, Behav. Neural Biol. 46, 137.

Van Wimersma Greidanus, Tj.B., F. Van de Brug, F. De Bruijckere, P.H.M.A. Pabst, R.W. Ruesink, R.L.E. Hulshof, B.N.M. Van Berckel, S.M. Arissen, E.J.P. De Koning and D.K. Donker, Comparison of bombesin, ACTH and $\beta$-endorphin induced grooming: Antagonism by haloperidol, naloxone and neurotensin, Ann. N.Y. Acad. Sci. (in press).

Vécsei, L., M. Balász and G. Telegdy, 1987, Action of somatostatin on the central nervous system, in: Neuropeptides and Brain Function, ed. G. Telegdy (Karger AG, Basel) p. 36. 